\title{
Anemia and Its Associated Risk Factors at the Time of Antiretroviral Therapy Initiation in Public Health Facilities of Arba Minch Town, Southern Ethiopia
}

\author{
Andamlak Gizaw Alamdo1, Temesgen Fiseha², Amanuel Tesfay ${ }^{3,4}$, Mesfin Kote Deber5, \\ Zemedu Mehamed Tirfe ${ }^{5}$, Tizta Tilahun 6 \\ ${ }^{1}$ Department of Public Nursing, Arba Minch Health Sciences College, Arba Minch, Ethiopia \\ ${ }^{2}$ Mizan Amman General Hospital, Southwest, Ethiopia \\ ${ }^{3}$ Department of Population and Family Health, College of Public Health and Medical Sciences, Jimma University, \\ Jimma, Ethiopia \\ ${ }^{4} \mathrm{CIH}$ LMU Center for International Health, Ludwig-Maximilians-Universitat, Munich, Germany \\ ${ }^{5}$ Epidemiology and Biostatstics Unit, Department of Public Health, College of Medicine and Health Sciences, \\ Arba Minch University, Arba Minch, Ethiopia \\ ${ }^{6}$ Population and Gender Unit, Institute of Development and Policy Research, Addis Ababa University, \\ Addis Ababa, Ethiopia \\ Email: gizandal@gmail.com, temesgen.fiseha@yahoo.com, amanuelpraise@yahoo.com,messi.kid@gmail.com, \\ zemedumohamed@yahoo.com, zemedu2003@gmail.com, tizta.tilahun@ju.edu.et
}

Received 5 August 2015; accepted 12 December 2015; published 15 December 2015

Copyright @ 2015 by authors and Scientific Research Publishing Inc.

This work is licensed under the Creative Commons Attribution International License (CC BY).

http://creativecommons.org/licenses/by/4.0/

(c) (i) Open Access

\section{Abstract}

Background: Anemia is the most common hematologic abnormality in HIV patients and is associated with disease progression and decreased survival. This study aims to describe the prevalence and predictors of anemia in HIV positive patients at the time of ART initiation at public health facilities of Arba Minch town, Southern Ethiopia. Methods: A total of 411 adults ( $\geq 15$ years) HIV positive patients with complete information on hemoglobin levels and CD4 count and clinical characteristics registered from 2006 to 2013 were assessed for anemia prevalence and risk factors at the ART clinic of the Arba Minch hospital and health center. The measurements of Hemoglobin and CD4 + T cell count were performed using standard methodology at baseline of ART initiation. Results: A total of $411 \mathrm{HIV}$ positive patients (195 males and 216 females) with a mean \pm SD age of $33.9 \pm 9.0$ years were assessed. Hemoglobin levels were between 6.0 and $16.5 \mathrm{mg} / \mathrm{dL}$. The overall prevalence of anemia at the time of ART initiation was $52.3 \%$; with $28.1 \%, 22.9 \%$ and $1.3 \%$ mild, moderate and severe anemia respectively. The overall prevalence of anemia was $62.4 \%$ 
among males and $46.7 \%$ among females $(P<0.001)$. An increased risk of anemia was seen in males (adjusted $\mathrm{OR}=2.78,95 \% \mathrm{CI}=1.77-4.35$ ); low $\mathrm{CD} 4$ cell counts (adjusted $\mathrm{OR}=3.48,95 \% \mathrm{CI}=$ 2.09 - 5.79); and history of TB (adjusted $\mathrm{OR}=2.89,95 \% \mathrm{CI}=1.28-6.54)$. Conclusions: Anemia in HIV-positive patients was highly prevalent at the time of ART initiation. Male gender, low CD4 count and history of TB were associated with higher risk of baseline anemia.

\section{Keywords}

\section{Prevalence, Antiretroviral Therapy (ART), Anemia, Predictors}

\section{Introduction}

Infection by the human immunodeficiency virus (HIV) and the consequent fully developed AIDS can have haematological effects, not only in patients with advanced disease but also during primary infection and the phase of clinical latency [1]. Hhematologic abnormalities that may be attributed to effects of HIV infection itself, to opportunistic infections or as a consequence of therapeutic agents are common manifestations causing symptoms that are life-threatening and have been documented as strong independent predictors of morbidity and mortality in HIV-infected individuals [2] [3].

Anemia is recognized to be the most common hematological abnormalities in patients with HIV infection [4] and is an important clinical problem in these patients both before and after the advent of combination antiretroviral therapy (ART) [5]. Anemia in HIV infected patients can have serious implications, which vary from functional and quality-of-life decrements to an association with disease progression. Evidences indicate that anemia among these patients is associated with a much faster rate of disease progression and decreases quality of life, and is a strong prognostic marker for death [1] [6] [7]. As recovery from anemia reduces the risk of disease progression at the approximately same level as seen among patients who have never had anemia; better understanding of base line anemia in HIV positive population will be critical not only in improving quality of life, but also in suggesting ways to reduce mortality [5] [8].

Depending on the study setting, anemia can be found in $63 \%$ - $95 \%$ of those with HIV infection at some point during the course of their disease which may be caused by a wide range of etiologic factors leading to decreased red blood cell (RBC) production, increased RBC destruction, or ineffective RBC production [2] [9]. Recent study reports a considerably higher prevalence of anemia among HIV-infected antiretroviral-naïve adult Ethiopians, with 38\% for men and 62\% for women [10]. However, the magnitude of anemia and its risk factors among HIV patients on initiating ART are not well document in Ethiopia. Thus, the aim of this study is to determine the prevalence of anemia and related factors among HIV infected patients at the time of ART initiation at ART clinic of public health facilities of Arba-Minch town, Southern Ethiopia.

\section{Materials and Methods}

The study was conducted in the hospital and health center of Arba Minch town, Gamo Gofa zone, Southern Ethiopia.

The hospital serves as a referral center for the surrounding zones and is located in the Southwestern part of Ethiopia. Arba Minch hospital and Arba Minch health center provides HIV/AIDS interventions, including free diagnosis, treatment and monitoring. The center diagnoses new cases and monitors those on therapy; consisting the HAART regimen Zidovudine, Stavudine and Nevirapine.

The data source for study variable was both medical and electronic records of patients kept in the health facilities. The medical and electronic records of each individual have a unique patient identifier that can be used to collect the history of medical care utilization for each individual.

These documents contain information on age, gender, date of HIV screening and ART initiation, history of opportunistic infections (OIs) and concurrent medical conditions, immunological responses, and therapy-related to prevention of mother to child transmission of HIV, ARV drugs, Cotrimoxazole and INH prophylaxis. Data abstraction form was used to retrieve relevant data from medical records of patients.

About 4656 medical records of ART patients initiating HAART between February 1st, 2006 and January 30th, 
2013, were evaluated to select eligible study subjects in the public health facilities of the Arba Minch town. Among this; 1952 (41.9\%) were excluded from the study for the following reasons. One hundred and eighty five (9.4\%) patients had age less than 15 years, 1724 (88.3\%) were excluded due to they were on pre-ART, and the remaining 43 (2.2\%) were pregnant at the time of ART initiation.

The sample size was determined by using a formula for estimation of single population proportion with the assumption of $95 \%$ confidence level, the margin of error of $5 \%$ and by taking the prevalence of anemia (50\%) (There was no previous research conducted in the district). After considering $10 \%$ contingency, the total sample size was estimated to be 422. Simple random sampling technique was employed to select samples using computer-generated random number table. Out of 422 study subjects; 411 HIV/AIDS patients' data were recruited for this study (11 Patients had incompletely recorded at baseline were excluded).

Before collection of the data, a data extraction tool was prepared and pre tested. Additionally, the nurses who were selected to collect the data were given one day training on the data extraction tool and how to collect data from registers to ensure no selection or information bias was introduced at the time of data collection. During data collection, the principal investigator supervises the data collectors and examined the tools for accuracy and completeness.

SPSS version 20 statistical software was used for performing statistical analyses and results are presented as number and percentage or mean \pm SD. Student's $t$ test and chi-squares were used for comparison of means and proportions, where appropriate. The crude (unadjusted) relationships between the exposure variables and the presence or absence of anemia were examined in univariate logistic regression. Multivariate logistic regression was used to identify the independent predictors of anemia. Anemia was defined according to the WHO criteria: $\mathrm{Hb}$ concentration $<13 \mathrm{~g} / \mathrm{dl}$ for males and $<12 \mathrm{~g} / \mathrm{dl}$ for females. We classified anemia as mild (11 - 12.9 for males and $11-11.9 \mathrm{~g} / \mathrm{dl}$ for females) moderate $(8-10 \mathrm{~g} / \mathrm{dl})$ and sever $(<8 \mathrm{~g} / \mathrm{dl})$.

Permission to undertake the study was obtained from the ethical review committee of Jimma University. The official letter of cooperation was written to Arba Minch Hospital and Health center, and permission from the ART clinic of the hospital and health center, where the data collection took place was obtained. Confidentiality of the patient information was strict and no information was handed over to a third party.

\section{Results}

Out of 422 study subjects, 411 HIV positive individuals (195 males and 216 females) were assessed for the presence of anemia at the time of ART initiation at the ART clinic of the Arba Minch hospital and health center. The mean \pm SD age of the study participants was $33.9 \pm 9.0$ years, and $76.3 \%$ of them were 40 years old or less. Of the study participants, 323 (83.5\%) were urban residents, 274 (71.4\%) had less than a high school education, and $233(60.7 \%)$ were married. The median \pm SD CD4 count of the study participants was $176.00 \pm 87.18$ cells $/ \mathrm{mm}^{3}$. Only, 136 (35.4\%), 39 (10.2\%) and 26 (6.8\%) of study participants had a positive history of opportunistic infections, tuberculosis (TB) and use of medications at the time of ART initiation (Table 1).

Anemia was present in $52.3 \%$ of the study participants, with $28.1 \%, 22.9 \%$ and $1.3 \%$ described as mild, moderate and severe anemia respectively. The overall prevalence of anemia was $62.4 \%$ among males and $46.7 \%$ among females. Gender had a significant influence on the overall prevalence of anemia $(P=0.002)$ and of mild anemia $(P<0.001)$, but not on the prevalence of moderate and severe anemia at the time of ART initiation $(P=$ 0.333 and $P=0.271$ ).

The prevalence of anemia was 53.5\%, 53.6\%, and 53.8\% among patients whose age group was within $<30$, $30-40$, and $<40$ years, respectively. The prevalence of overall anemia as well as mild, moderate and severe anemia did not differ significantly according to age (all $P>0.05$ ). Additionally, no significant difference was observed in the prevalence of anemia with educational status and marital status of participants (all $P>0.05$ ).

Based on baseline CD4 count, there was a significant difference in the prevalence of anemia within the three CD4 categories, with $60.5 \%$ among patients with CD4 count $<200$ cells $/ \mathrm{mm}^{3}$, and $53.3 \%$ and $37.8 \%$ among those with CD4 count of $>350$ and $200-350$ cells $/ \mathrm{mm}^{3}$ respectively $(P<0.001)$. The prevalence of moderate anemia increased with decreasing CD4 count $(P=0.001)$, while prevalence of mild anemia, and of severe anemia did not increase with decreasing CD4 count $(P=0.377$ and $P=0.794)$.

The overall prevalence of anemia was significantly higher among patients with history of TB $(73.2 \%$ vs. 49.9\%; $P=0.005)$, use of medications $(76.9 \%$ vs. $50.4 \% ; P=0.009)$, and opportunistic infections (59.6\% vs. 48.2\%; $P=0.033$ ). The prevalence of moderate anemia, and of mild anemia was significantly higher among patients with history of TB and use of medications $(P=0.009$ and $P=0.003)$, and opportunistic infections at the 
Table 1. Baseline characteristics of HIV-Infected adults at the time of ART initiation in public health facilities of ArbaMinch town, Southern Ethiopia, $2013(n=411)$.

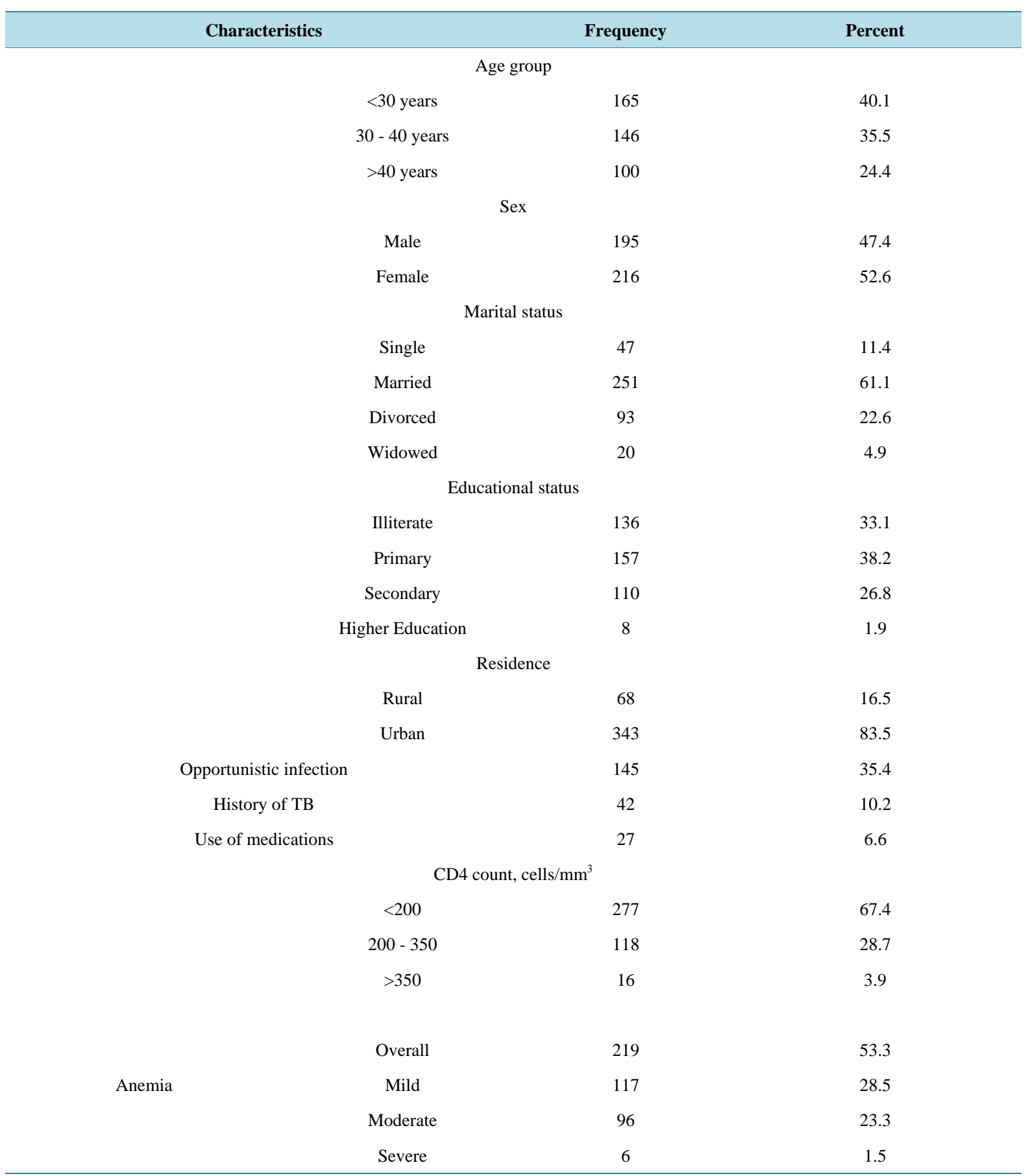

time of ART initiation $(P=0.022)$. The prevalence of all forms of anemia did not differ significantly by residence and functional status of patients $(P=0.090$ and $P=0.376)$ (Table 2$)$.

In a multivariate analysis using a logistic regression model, we analyzed factors associated with the presence of anemia. Table 3 summarizes the results of the final regression model. In this study sex, CD4 count and past TB treatment were significantly associated to anemia. The odds of anemia among male participants was 2.8 times $(\mathrm{OR}=2.78,95 \% \mathrm{CI}=1.77$ - 4.35) compared to the odds among female counter parts. The odds of anemia among individuals with baseline low CD4 count $\left(<200\right.$ cells $\left./ \mathrm{mm}^{3}\right)$ were higher $(\mathrm{OR}=3.48,95 \% \mathrm{CI}=2.09-$ 
5.79) compared to those participants with high CD4 count. The odds of anemia among participants having history of TB were higher $(\mathrm{OR}=2.89,95 \% \mathrm{CI}=1.28-6.54)$ compared to odds of those without history of TB (Table 3).

\section{Discussion}

Anemia is a frequent complication of HIV infection that is associated with an increased risk of death and that recovery from anemia is associated with decreased risk of death for HIV-infected persons who do develop anemia [8]. As recovery from anemia increase survival, screening for anemia among HIV positive population at base line should be aggressive and critical not only to reduce mortality, but also in suggesting ways to improve quality of life [5] [8].

The prevalence of anemia in this study was $52.3 \%$ which is in agreement with the study in China (51.9\%) [11] and in Nigeria (57.5\%) [12], but lower than that found by others in the country which reported $86.5 \%$ prevalence of anemia at base line [13]. However, our prevalence estimate was higher than other anemia prevalence studies in Northwest Ethiopia [10] and in the USA [14], which reported 35\% and 39.7\% prevalence of anemia at baseline among HIV patients respectively.

Table 2. Distribution of overall prevalence of Anemia among HIV-Infected adults in public health facilities of Arba-Minch town, Southern Ethiopia, $2013(n=411)$.

\begin{tabular}{|c|c|c|c|}
\hline Variables & & N (\%) & $P$-value \\
\hline \multicolumn{4}{|l|}{ Age } \\
\hline & $\leq 40$ years & $48(52.7)$ & \multirow{2}{*}{0.930} \\
\hline & $>40$ years & $153(52.2)$ & \\
\hline \multicolumn{4}{|c|}{ Sex } \\
\hline & Male & 107 (62.9) & \multirow{2}{*}{$<0.001$} \\
\hline & Female & 94 (43.9) & \\
\hline \multicolumn{4}{|c|}{ Education } \\
\hline & $<$ High school & $152(55.5)$ & \multirow{2}{*}{0.053} \\
\hline & $\geq$ High school & $49(44.5)$ & \\
\hline \multicolumn{4}{|c|}{ Residence } \\
\hline & Rural & 38 (62.3) & \multirow{2}{*}{0.090} \\
\hline & Urban & $163(50.5)$ & \\
\hline \multicolumn{4}{|c|}{ History of TB } \\
\hline & Yes & $30(73.2)$ & \multirow{2}{*}{0.005} \\
\hline & No & $171(49.9)$ & \\
\hline \multicolumn{4}{|c|}{ Opportunistic infection } \\
\hline & Yes & $81(59.6)$ & \multirow{2}{*}{0.033} \\
\hline & No & $119(48.2)$ & \\
\hline \multicolumn{4}{|c|}{ Use of medications } \\
\hline & Yes & 20 (76.9) & \multirow{2}{*}{0.009} \\
\hline & No & $180(50.4)$ & \\
\hline \multicolumn{4}{|c|}{ Functional status } \\
\hline & Working & 169 (52.6) & \multirow{2}{*}{0.376} \\
\hline & Ambulatory/Bed ridden & 37 (58.7) & \\
\hline \multicolumn{4}{|c|}{ CD4 count } \\
\hline & $<200$ cells $/ \mathrm{mm}^{3}$ & $152(58.9)$ & \multirow{2}{*}{$<0.001$} \\
\hline & $\geq 200$ cells $/ \mathrm{mm}^{3}$ & 49 (38.9) & \\
\hline
\end{tabular}


Table 3. Predictors of Anemia among HIV-infected adults in public health facilities of Arba-Minch town, Southern Ethiopia, $2013(n=411)$.

\begin{tabular}{|c|c|c|c|}
\hline Variables & & Adjusted OR (95\% CI) & $P$-value \\
\hline & \multicolumn{2}{|c|}{ Sex } & \multirow{3}{*}{$<0.001$} \\
\hline & Male & $2.78(1.77-4.35)$ & \\
\hline & Female & 1 & \\
\hline & \multicolumn{2}{|c|}{ Education } & \\
\hline & $<$ High school & $1.53(0.96-2.45)$ & \multirow{2}{*}{0.080} \\
\hline & $\geq$ High school & 1 & \\
\hline & \multicolumn{2}{|c|}{ History of TB } & \multirow{3}{*}{0.022} \\
\hline & Yes & $2.89(1.28-6.54)$ & \\
\hline & No & 1 & \\
\hline & \multicolumn{2}{|c|}{ Opportunistic infection } & \multirow{3}{*}{0.091} \\
\hline & Yes & $1.47(0.97-2.21)$ & \\
\hline & No & 1 & \\
\hline & \multicolumn{2}{|c|}{ Use of medications } & \\
\hline & Yes & $2.74(0.94-7.97)$ & \multirow{2}{*}{0.064} \\
\hline & No & 1 & \\
\hline & \multicolumn{2}{|c|}{ Residence } & \multirow{3}{*}{0.054} \\
\hline & Rural & $1.95(0.99-3.51)$ & \\
\hline & Urban & 1 & \\
\hline & \multicolumn{2}{|c|}{ CD4 count } & \\
\hline & $<200$ cells $/ \mathrm{mm}^{3}$ & $3.48(2.09-5.79)$ & \multirow{2}{*}{$<0.001$} \\
\hline & $\geq 200$ cells $/ \mathrm{mm}^{3}$ & & \\
\hline
\end{tabular}

In this study, male HIV patients were found to have a significantly higher prevalence of anemia than females: $62.4 \%$ vs. $46.7 \%(P<0.001)$. This finding was in agreement with findings of the USA study [14], but in contrast with the findings of studies in Northwest Ethiopia and China [10] [11]. However, the overall prevalence of anemia in our study population did not differ significantly according to age. Thus our finding is not inconsistent with the China study finding, which reported older age significantly associated with an increased risk of anemia [11].

In our study, the prevalence of anemia increased with decreasing CD4 count $(P<0.001)$ with pick prevalence of among patients with CD4 count $<200$ cells $/ \mathrm{mm}^{3}$. This finding was consistent with several published studies, which reported that anemia was more prevalent among patients with CD4 count $<200$ cells $/ \mathrm{mm}^{3}$ [11] [14]. Mostly, prevalence of anemia is higher among patients who have low CD4 count, which was also reported by others in the country [10]. In general, the likelihood of anemia increases with progressive immunologic deterioration and a CD4 count less than 200 cells/ $\mathrm{mm}^{3}$ is associated with the development of anemia; in which HIV infected patients with AIDS were more likely to develop anemia compared with those without AIDS.

Other independent predictor of anemia in this study was history of TB; patients with history of TB have higher risk of developing anemia than those without TB. Various studies have found that hemoglobin levels among TB-HIV co-infected individuals were considerably lower than among subjects with only one of the two infections [15] [16]. Various pathogeneses have been suggested in TB-associated anemia, studies have shown suppression of erythropoiesis by inflammatory mediators as a cause of anemia. 
To the best of our knowledge, this is the first study in our country Ethiopia to assess the prevalence of anemia among HIV infected patients at the time of ART initiation. However, this study had limitations such as data were collected for routine clinical care, and hence, other important variables, notably erythropoietin levels and viral load are lacking. In addition, the majority of patients had no data on BMI. Farther longitudinal studies should be conducted to develop interventions aimed at reducing the prevalence of anemia in HIV infected patients.

\section{Conclusion}

Anemia is highly prevalent among HIV-infected adults in public health facilities of Arba-Minch town, Southern Ethiopia at the time of ART initiation. Male gender, lower CD4 count and history of TB disease are associated with an increased risk of baseline anemia. It is important to routinely screen anemia not only for reducing mortality, but also for suggesting ways to improve quality of life of these patient. Further research is needed to evaluate the effects of treating anemia and the impact of anemia on ART and survival in HIV-infected patients in this area.

\section{Acknowledgements}

We are grateful to acknowledge Jimma University College of Public Health and Medical Sciences for providing financial assistance for the study. We also like to acknowledge data collectors and administrations of the Arba-Minch hospital and health center.

\section{Competing Interests}

'The authors declare that they have no competing interests.

\section{Authors' Contributions}

AG conceived of the study, and participated in its design and statistical analysis and helped to draft the manuscript; TT; TF and AT participated in design and statistical analysis of the study, MK and ZM contributed in statistical analysis and helped to draft the manuscript. All authors read and approved the final manuscript.

\section{References}

[1] Bain, B.J. (1997) The Haematological Features of HIV Infection. Review. British Journal of Haematology, 99, 1-8. http://dx.doi.org/10.1046/j.1365-2141.1997.2943111.x

[2] Mehta, S., Jutur, S. and Gautam, D. (2011) Hematologic Manifestations of HIV/AIDS. Medicine Update-2011.

[3] Volberding, P., Baker, K. and Levine, A. (2003) Human Immunodeficiency Virus Hematology. Hematology Am Soc Hematol Educ Program, 2003, 294-313. http://dx.doi.org/10.1182/asheducation-2003.1.294

[4] Sharma, S. (2014) Normocytic Norm Chromic Anemia Is Most Common Type Anemia HIV Infected Patients. JMSCR, 2, 1249-1255.

[5] Amanda, J. and Nancy Berliner, R. (2013) Pathogenesis and Clinical Implications of HIV-Related Anemia in 2013. Hematology, the Education Program, 2013, 377-381. http://dx.doi.org/10.1182/asheducation-2013.1.377

[6] Belperio, P. and Rhew, D. (2004) Prevalence and Outcomes of Anemia in Individuals with Human Immunodeficiency Virus: A Systematic Review of the Literature. American Journal of Medicine, 116, 27-43. http://dx.doi.org/10.1016/j.amjmed.2003.12.010

[7] Mocroft, A., Kirk, O., Barton, S.E., Dietrich, M., Proenca, R., Colebunders, R., et al. (1999) Anaemia Is an Independent Predictive Marker for Clinical Prognosis in HIV-Infected Patients from across Europe. AIDS, 13, 943-450. http://dx.doi.org/10.1097/00002030-199905280-00010

[8] Sullivan, P., Hanson, D., Chu, S., Jones, J. and Ward, J. (1998) Epidemiology of Anemia in Human Immunodeficiency Virus (HIV)-Infected Persons: Results from the Multistate Adult and Adolescent Spectrum of HIV Disease Surveillance Project. Blood, 91, 301-308.

[9] Obirikorang, C. and Yeboah, F. (2009) Blood Hemoglobin Measurements as a Predictive Indicator for the Progression of HIV/AIDS in Resource-Limited Setting. Journal of Biomedical Science, 16, 102. http://dx.doi.org/10.1186/1423-0127-16-102

[10] Ferede, G. and Wondimeneh, Y. (2013) Prevalence and Related Factors of Anemia in HAART-Naive HIV Positive Pa- 
tients at Gondar University Hospital, Northwest Ethiopia. BMC Hematology, 13, 8. http://dx.doi.org/10.1186/2052-1839-13-8

[11] Shen, Y., Wang, Z., Lu, H., Wang, J., Chen, J., Liu, L., et al. (2013) Prevalence of Anemia among Adults with Newly Diagnosed HIV/AIDS in China. PLoS ONE, 8, e73807. http://dx.doi.org/10.1371/journal.pone.0073807

[12] Denue, B., Kida, I., Hammagabdo, A., Dayar, A. and Sahabi, M. (2013) Prevalence of Anemia and Immunological Markers in HIV-Infected Patients on Highly Active Antiretroviral Therapy in Northeastern Nigeria. Infectious Diseases: Research and Treatment, 6, 25-33. http://dx.doi.org/10.4137/IDRT.S10477

[13] Daka, D., Lelissa, D. and Amsalu, A. (2013) Prevalence of Anaemia before and after the Initiation of Antiretroviral Therapy at ART Centre of Hawassa University Referral Hospital, Hawassa, South Ethiopia. Sch J Med., 3, 1-6.

[14] Mildvan, D., Creagh, T. and Leitz, G., Anemia Prevalence Study Group (2007) Prevalence of Anemia and Correlation with Biomarkers and Specific Antiretroviral Regimens in 9690 Human-Immunodeficiency-Virus-Infected Patients: Findings of the Anemia Prevalence Study. Current Medical Research and Opinion, 23, 343-355. http://dx.doi.org/10.1185/030079906X162683

[15] Lawson, L., Yassin, M.A., Thacher, T.D., Olatunji, O.O., Lawson, J.O., Akingbogun, T.I., et al. (2008) Clinical Presentation of Adults with Pulmonary Tuberculosis with and without HIV Infection in Nigeria. Scandinavian Journal of Infectious Diseases, 40, 30-35. http://dx.doi.org/10.1080/00365540701509899

[16] Whalen, C.C., Nsubuga, P., Okwera, A., Johnson, J.L., Hom, D.L., Michael, N.L., et al. (2000) Impact of Pulmonary Tuberculosis on Survival of HIV-Infected Adults: A Prospective Epidemiologic Study in Uganda. AIDS, 14, 12191228. 